Article

\title{
On a New Formula for Fibonacci's Family m-step Numbers and Some Applications
}

\author{
Monther Rashed Alfuraidan *(D) and Ibrahim Nabeel Joudah * \\ Department of Mathematics \& Statistics, King Fahd University of Petroleum and Minerals, Dhahran 31261, \\ Saudi Arabia \\ * Correspondence: monther@kfupm.edu.sa (M.R.A.); ibrahim.inj@gmail.com (I.N.J.)
}

Received: 6 July 2019; Accepted: 27 August 2019; Published: 1 September 2019

check for updates

\begin{abstract}
In this work, we obtain a new formula for Fibonacci's family $m$-step sequences. We use our formula to find the $n$th term with less time complexity than the matrix multiplication method. Then, we extend our results for all linear homogeneous recurrence $m$-step relations with constant coefficients by using the last few terms of its corresponding Fibonacci's family $m$-step sequence. As a computational number theory application, we develop a method to estimate the square roots.
\end{abstract}

Keywords: Fibonacci's $m$-step numbers; time complexity; linear homogeneous recurrence relations

AMS Subject Classification: 11B37; 11B39; $11 \mathrm{Y} 16$

\section{Introduction}

Currently, in modern science, extensive work has been done in the area of recurrence relations and their applications (see, e.g., [1-5]).

In $[6,7]$, the authors developed a transformation method of Tribonacci sequence and Tetranacci sequence to find the $n$th term of any Tribonacci-Like sequence and Tetranacci-Like sequence, respectively. In [8], the authors extended the previous transformation method to any Fibonacci-Like $m$-step sequence.

In [9], the authors used matrix multiplication to find the $n$th term of Fibonacci's family $m$-step sequences. The time complexity of their result is of order $m^{3} \log n$ times the time of multiplying two n-digit integers.

We generalize the transformation methods in [6,7] for Fibonacci's family $m$-step sequences. We also use matrix method as in [9] to obtain the closed form of our new formula. However, the time complexity of our formula is of order $m^{2} \log n$ times the time of multiplying two $n$-digit integers. As a computational number theory application, we develop a method to estimate the square root. The paper is organized as follows:

- Section 2 contains the notations and definitions related to this work. Section 2.1 gives a look into Fibonacci sequences and their properties. One of the most important features linked to the evaluation of iterative methods is the order of convergence or the time complexity. Time complexity shows how fast the algorithm converges to the solution. This aspect is discussed briefly in Section 2.2.

- Section 3 provides the main results and is organized as follows. In Section 3.1, we state and prove our main results. In Section 3.2, we provide a method of finding the $n$th term of any linear homogeneous recurrence relation. In Section 3.3, we illustrate our method by a numerical example.

- Section 4 deals with the computational number theory application; in particular, we give a different method of approximating the square roots. 


\section{Definitions and Notations}

\subsection{Fibonacci Primer}

The Fibonacci sequence shows a certain numerical pattern. This pattern turns out to have an interest and importance far beyond what its inventor imagined. It can be used to model or describe an amazing variety of phenomena, in mathematics, science, and art (see, e.g., [3,8]).

The well-known Fibonacci sequence of numbers which are defined by the recurrence

$$
F_{n}=F_{n-1}+F_{n-2}, n \geq 2
$$

with the initial values $F_{0}=0$ and $F_{1}=1$, is an example of a linear homogeneous recurrence sequence.

Definition 1. The linear homogeneous recurrence $m$-step sequence $\left\{H_{n}\right\}$, for $m \geq 2$ an arbitrary integer, is defined by the recurrence

$$
H_{n}=k_{1} H_{n-1}+k_{2} H_{n-2}+\cdots+k_{m} H_{n-m}, \quad n \geq m+1,
$$

where $k_{1}, \cdots, k_{m}$ are constants and $H_{1}, \cdots, H_{m}$ are the initial values.

Miles [4] appears to be the first who studied such sequences, with constants $k_{i}=1,1 \leq i \leq m$ and initial values $H_{1}=0, H_{2}=0, \cdots, H_{m-1}=0, H_{m}=1$.

Definition 2. The Fibonacci $m$-step sequence, for $m \geq 2$ an arbitrary fixed integer, is defined by the recurrence

$$
U_{n}=U_{n-1}+U_{n-2}+\cdots+U_{n-m}, n \geq 2,
$$

with the initial values $U_{2-m}=U_{1-m}=\cdots=U_{0}=0$, and $U_{1}=1$.

Definition 3. The Fibonacci family m-step sequence, for $m \geq 2$ an arbitrary fixed integer, is defined by the recurrence

$$
U_{n}=k_{1} U_{n-1}+k_{2} U_{n-2}+\cdots+k_{m} U_{n-m}, n \geq 2,
$$

with the initial values $U_{2-m}=U_{1-m}=\cdots=U_{0}=0$, and $U_{1}=1$.

Definition 4. The characteristic equation of Fibonacci m-step sequence is

$$
x^{m}-x^{m-1}-\cdots-x-1=0 .
$$

It is well known that such sequence has the following property:

Property 1. $\lim _{n \rightarrow \infty} \frac{U_{n+1}}{U_{n}}$ is equal to the leading root of Equation (3).

\subsection{The Time Complexity}

In [9], the authors used matrix multiplication to find the $n$th term of Fibonacci's family $m$-step sequences. The time complexity of their method is $O\left(m^{3} \times \log (n) \times M(n \times n)\right)$, where $M(n \times n)$ denotes the time of multiplying two $n$-digit integers. We also use matrix notation to obtain the closed form of our formula. However, the time complexity of this new formula is $O\left(m^{2} \times \log (n) \times\right.$ $M(n \times n))$. Notice that, in calculating the time complexity of iterative processes, it is often assumed that the arithmetic operations of addition and multiplication can be computed in constant times. This assumption is invalid if the number of digits depends on the index of the term $n$ as the computation proceeds. Therefore, it is important to distinguish between the process of multiplying two terms and term by a constant. Our actual time complexity is $O\left(m^{3} \times \log (1 \times n) \times M(1 \times n)+m^{2} \times \log (n) \times\right.$ 
$M(n \times n))$, based on the assumption that the terms of the sequence are integer numbers. As $m<<n$, our time complexity can be consider as $O\left(m^{2} \times \log (n) \times M(n \times n)\right)$.

\section{Main Results}

Let $H_{n}=\sum_{i=1}^{m} k_{i} H_{n-i}$, where $n \geq m+1$, be a linear homogenous recurrence $m$-step relation with constant coefficients $k_{i}, 1 \leq i \leq m$, and initial values $H_{1}, H_{2}, \cdots, H_{m}$. Let $U_{n}=\sum_{i=1}^{m} k_{i} U_{n-i}$, where $m \geq 2$, be a linear recurrence $m$-step relation with $U_{i}=0$, for $2-m \leq i \leq 0$ and $U_{1}=1$, i.e., $\left\{U_{n}\right\}$ is a Fibonacci family $m$-step sequence. We define the matrices $A_{m \times m}, K_{m \times m},{ }^{t} H_{1 \times m},{ }^{t} U_{1 \times m}$ as follows:

$$
\begin{aligned}
A_{m \times m}:= & \left(\begin{array}{ccccc}
k_{1} & k_{2} & k_{3} & \cdots & k_{m} \\
1 & 0 & 0 & \cdots & 0 \\
0 & 1 & 0 & \cdots & 0 \\
\vdots & \vdots & \vdots & \ddots & \vdots \\
0 & 0 & 1 & \cdots & 0
\end{array}\right), K_{m \times m}:=\left(\begin{array}{ccccc}
k_{1} & k_{2} & \cdots & k_{m-1} & k_{m} \\
k_{2} & k_{3} & \cdots & k_{m} & 0 \\
k_{3} & k_{4} & \cdots & 0 & 0 \\
\vdots & \vdots & \ddots & \vdots & \vdots \\
k_{m} & 0 & \cdots & 0 & 0
\end{array}\right) \\
{ }^{t} H_{1 \times m} & :=\left[\begin{array}{cccc}
H_{t} & H_{t-1} & \cdots & H_{t-m+1}
\end{array}\right],{ }^{t} U_{1 \times m}:=\left[\begin{array}{llll}
U_{t} & U_{t-1} & \cdots & U_{t-m+1}
\end{array}\right] .
\end{aligned}
$$

The following technical proposition are used to prove our main result.

Proposition 1. For positive integers $n, m, t$ with $n \geq m+1$, we have

$$
H_{n}=\sum_{i=1}^{m}\left(H_{n-i+1-t} \sum_{j=i}^{m} k_{j} U_{t+i-j}\right)
$$

Alternately, $H_{n}={ }^{(n-t)} H_{1 \times m} \times K_{m \times m} \times{ }^{t} U_{1 \times m}^{T}$, where ${ }^{t} U^{T}$ denotes the transpose of the matrix ${ }^{t} U$.

Proof. Let $H_{n}=\sum_{i=1}^{m} L_{i, t} H_{n-i+2-t}$, where $L_{i, t}$ is the coefficient of $H_{n-i+2-t}$. The following table shows that

$$
U_{t}=L_{1, t}, \text { for } 2-m \leq t \leq 1 .
$$

\begin{tabular}{|c|c|c|}
\hline$t$ & $\sum_{i=1}^{m} L_{i, t} H_{n-i+2-t}$ & $L_{1, t}$ \\
\hline 1 & $\sum_{i=1}^{m} L_{i, 1} H_{n-i+1}=(1) H_{n}+\sum_{i=1}^{m-1}(0) H_{n-i}$ & $L_{1,1}=1$ \\
\hline 0 & $\sum_{i=1}^{m} L_{i, 0} H_{n-i+2}=(0) H_{n+1}+(1) H_{n}+\sum_{i=1}^{m-2}(0) H_{n-i}$ & $L_{1,0}=0$ \\
\hline-1 & $\sum_{i=1}^{m} L_{i,-1} H_{n-i+3}=\sum_{i=1}^{2}(0) H_{n+3-i}+(1) H_{n}+\sum_{i=1}^{m-3}(0) H_{n-i}$ & $L_{1,-1}=0$ \\
\hline$\vdots$ & $\vdots$ & $\vdots$ \\
\hline $3-m$ & $\sum_{i=1}^{m} L_{i, 3-m} H_{n-i+m-1}=\sum_{i=1}^{m-2}(0) H_{n+m-1-i}+(1) H_{n}+(0) H_{n-1}$ & $L_{1,3-m}=0$ \\
\hline $2-m$ & $\sum_{i=1}^{m} L_{i, 2-m} H_{n-i+m}=\sum_{i=1}^{m-1}(0) H_{n+m-i}+(1) H_{n}$ & $L_{1,2-m}=0$ \\
\hline
\end{tabular}


Now,

$$
\begin{aligned}
H_{n} & =\sum_{i=1}^{m} L_{i, t} H_{n-i+2-t} \\
& =L_{1, t} H_{n+1-t}+\sum_{i=2}^{m} L_{i, t} H_{n-i+2-t} \\
& =L_{1, t} \sum_{i=1}^{m} k_{i} H_{n+1-t-i}+\sum_{i=2}^{m} L_{i, t} H_{n-i+2-t} \\
& =\sum_{i=1}^{m} k_{i} L_{1, t} H_{n+1-t-i}+\sum_{i=1}^{m-1} L_{i+1, t} H_{n-i+1-t} \\
& =\sum_{i=1}^{m-1}\left(k_{i} L_{1, t}+L_{i+1, t}\right) H_{n-i+1-t}+k_{m} L_{1, t} H_{n-m+1-t} \\
& =\sum_{i=1}^{m-1}\left(k_{i} L_{1, t}+L_{i+1, t}\right) H_{n-i+2-(t+1)}+k_{m} L_{1, t} H_{n-m+2-(t+1)}
\end{aligned}
$$

Since $H_{n}=\sum_{i=1}^{m} L_{i, t+1} H_{n-i+2-(t+1)}=\sum_{i=1}^{m-1} L_{i, t+1} H_{n-i+2-(t+1)}+L_{m, t+1} H_{n-m+2-(t+1)}$, then from $\left(^{*}\right)$, we have $L_{m, t+1}=k_{m} L_{1, t}$ and

$$
L_{i, t+1}=k_{i} L_{1, t}+L_{i+1, t} \text { for } 1 \leqslant i \leqslant m-1
$$

By backward substitution in Equation (5), we have

$$
\begin{aligned}
L_{m-1, t} & =k_{m-1} L_{1, t-1}+k_{m} L_{1, t-2} \\
L_{m-2, t} & =k_{m-2} L_{1, t-1}+k_{m-1} L_{1, t-2}+k_{m} L_{1, t-3} \\
\vdots & \\
L_{i, t} & =k_{i} L_{1, t-1}+k_{i+1} L_{1, t-2}+\cdots+k_{m} L_{1, t+i-1-m}
\end{aligned}
$$

and hence,

$$
L_{i, t}=\sum_{j=i}^{m} k_{j} L_{1, t+i-1-j}
$$

Now, from Equations (4) and (6), we have $L_{1, t}=U_{t} \forall t$ and $L_{i, t}=\sum_{j=i}^{m} k_{j} U_{t+i-1-j}$. Thus,

$$
H_{n}=\sum_{i=1}^{m} L_{i, t} H_{n-i+2-t}=\sum_{i=1}^{m} H_{n-i+2-t} \sum_{j=i}^{m} k_{j} U_{t+i-1-j} .
$$

Now, by replacing $t$ by $t+1$, we get

$$
H_{n}=\sum_{i=1}^{m}\left(H_{n-i+1-t} \sum_{j=i}^{m} k_{j} U_{t+i-j}\right) .
$$

Alternately, $H_{n}={ }^{(n-t)} H_{1 \times m} \times K_{m \times m} \times{ }^{t} U_{1 \times m}^{T}$.

\subsection{Main Formulas}

Lemma 1. For positive integers $z, m, t$ with $z=2 n$ and $n \geq m+1$, we have

$$
H_{2 n+1}={ }^{2 n-t} H_{1 \times m} \times K_{m \times m} \times A_{m \times m} \times{ }^{t} U_{1 \times m}^{T}
$$

Proof. By Proposition 1, we have

$$
H_{2 n+b}={ }^{2 n+b-t} H_{1 \times m} \times K_{m \times m} \times{ }^{t} U_{1 \times m}^{T}
$$


In particular, we have

$$
H_{2 n+1}={ }^{2 n+1-t} H_{1 \times m} \times K_{m \times m} \times{ }^{t} U_{1 \times m}^{T} .
$$

Thus,

$$
\begin{aligned}
& H_{2 n+1}=\left(\begin{array}{c}
H_{2 n-t+1} \\
H_{2 n-t} \\
\vdots \\
H_{2 n-t-m+2}
\end{array}\right)^{T}\left(\begin{array}{cccc}
k_{1} & k_{2} & \cdots & k_{m} \\
k_{2} & k_{3} & \cdots & 0 \\
\vdots & \vdots & \ddots & \vdots \\
k_{m} & 0 & \cdots & 0
\end{array}\right)\left(\begin{array}{c}
U_{t} \\
U_{t-1} \\
\vdots \\
U_{t-m+1}
\end{array}\right) \\
& =\left(\begin{array}{c}
0 \\
H_{2 n-t} \\
\vdots \\
H_{2 n-t-m+2}
\end{array}\right)^{T}\left(\begin{array}{cccc}
0 & 0 & \cdots & 0 \\
k_{2} & k_{3} & \cdots & 0 \\
\vdots & \vdots & \ddots & \vdots \\
k_{m} & 0 & \cdots & 0
\end{array}\right)\left(\begin{array}{c}
U_{t} \\
U_{t-1} \\
\vdots \\
U_{t-m+1}
\end{array}\right) \\
& +H_{2 n-t+1}\left(\begin{array}{c}
k_{1} \\
k_{2} \\
\vdots \\
k_{m}
\end{array}\right)^{T}\left(\begin{array}{c}
U_{t} \\
U_{t-1} \\
\vdots \\
U_{t-m+1}
\end{array}\right) \\
& =\left(\begin{array}{c}
H_{2 n-t} \\
\vdots \\
H_{2 n-t-m+2} \\
0
\end{array}\right)^{T}\left(\begin{array}{cccc}
k_{2} & k_{3} & \cdots & 0 \\
\vdots & \vdots & \ddots & \vdots \\
k_{m} & 0 & \cdots & 0 \\
0 & 0 & \cdots & 0
\end{array}\right)\left(\begin{array}{c}
U_{t} \\
U_{t-1} \\
\vdots \\
U_{t-m+1}
\end{array}\right) \\
& +\left(\begin{array}{c}
H_{2 n-t} \\
\vdots \\
H_{2 n-t-m+2} \\
H_{2 n-t-m+1}
\end{array}\right)^{T}\left(\begin{array}{c}
k_{1} \\
k_{2} \\
\vdots \\
k_{m}
\end{array}\right)\left(\begin{array}{c}
k_{1} \\
k_{2} \\
\vdots \\
k_{m}
\end{array}\right)^{T}\left(\begin{array}{c}
U_{t} \\
U_{t-1} \\
\vdots \\
U_{t-m+1}
\end{array}\right) \\
& =\left(\begin{array}{c}
H_{2 n-t} \\
\vdots \\
H_{2 n-t-m+2} \\
H_{2 n-t-m+1}
\end{array}\right)^{T}\left(\begin{array}{cccc}
k_{2} & k_{3} & \cdots & 0 \\
\vdots & \vdots & \ddots & \vdots \\
k_{m} & 0 & \cdots & 0 \\
0 & 0 & \cdots & 0
\end{array}\right)\left(\begin{array}{c}
U_{t} \\
U_{t-1} \\
\vdots \\
U_{t-m+1}
\end{array}\right)
\end{aligned}
$$




$$
\begin{aligned}
& +\left(\begin{array}{c}
H_{2 n-t} \\
\vdots \\
H_{2 n-t-m+2} \\
H_{2 n-t-m+1}
\end{array}\right)^{T}\left(\begin{array}{cccc}
k_{1} k_{1} & \cdots & k_{m-1} k_{1} & k_{m} k_{1} \\
k_{1} k_{2} & \cdots & k_{m-1} k_{2} & k_{m} k_{2} \\
\vdots & \ddots & \vdots & \vdots \\
k_{1} k_{m} & \cdots & k_{m-1} k_{m} & k_{m} k_{m}
\end{array}\right)\left(\begin{array}{c}
U_{t} \\
U_{t-1} \\
\vdots \\
U_{t-m+1}
\end{array}\right) \\
& =\left(\begin{array}{c}
H_{2 n-t} \\
\vdots \\
H_{2 n-t-m+2} \\
H_{2 n-t-m+1}
\end{array}\right)^{T}\left(\begin{array}{cccc}
k_{1} k_{1}+k_{2} & \cdots & k_{m-1} k_{1}+k_{m} & k_{m} k_{1} \\
k_{1} k_{2}+k_{3} & \cdots & k_{m-1} k_{2} & k_{m} k_{2} \\
\vdots & \ddots & \vdots & \vdots \\
k_{1} k_{m} & \cdots & k_{m-1} k_{m} & k_{m} k_{m}
\end{array}\right)\left(\begin{array}{c}
U_{t} \\
U_{t-1} \\
\vdots \\
U_{t-m+1}
\end{array}\right) \\
& =\left(\begin{array}{c}
H_{2 n-t} \\
\vdots \\
H_{2 n-t-m+2} \\
H_{2 n-t-m+1}
\end{array}\right)^{T}\left(\begin{array}{cccc}
k_{1} & k_{2} & \cdots & k_{m} \\
k_{2} & k_{3} & \cdots & 0 \\
\vdots & \vdots & \ddots & \vdots \\
k_{m} & 0 & \cdots & 0
\end{array}\right)\left(\begin{array}{cccc}
k_{1} & k_{2} & \cdots & k_{m} \\
1 & 0 & \cdots & 0 \\
\vdots & \ddots & \ddots & \vdots \\
0 & \cdots & 1 & 0
\end{array}\right)\left(\begin{array}{c}
U_{t} \\
U_{t-1} \\
\vdots \\
U_{t-m+1}
\end{array}\right) \\
& ={ }^{2 n-t} H_{1 \times m} \times K_{m \times m} \times A_{m \times m} \times{ }^{t} U_{1 \times m}^{T} .
\end{aligned}
$$

The following two results are used to get our main formula of $U_{2 n}$ in terms of ${ }^{n} U_{1 \times m}$ only.

Theorem 1. For integers $m, n, t, b$ with $n>m>1$, we have

$$
H_{2 n+b}={ }^{2 n-t} H_{1 \times m} \times K_{m \times m} \times A_{m \times m}^{b} \times{ }^{t} U_{1 \times m}^{T},
$$

where ${ }^{t} U^{T}$ denotes the transpose of the matrix ${ }^{t} U$.

Proof. Lemma 1 gives the result for $b=1$. Now, assume the statement is true for $b-1$, i.e.,

$$
H_{2 n+b-1}={ }^{2 n-t} H_{1 \times m} \times K_{m \times m} \times A_{m \times m}^{b-1} \times{ }^{t} U_{1 \times m}^{T} .
$$

Replacing $n$ by $n+\frac{1}{2}$ in Equation (10), we get

$$
H_{2 n+b}={ }^{2 n-t+1} H_{1 \times m} \times K_{m \times m} \times A_{m \times m}^{b-1} \times{ }^{t} U_{1 \times m}^{T} .
$$

By repeating the same method of Lemma 1, one can easily show that

$$
H_{2 n+b}={ }^{2 n-t} H_{1 \times m} \times K_{m \times m} \times A_{m \times m}^{b} \times{ }^{t} U_{1 \times m}^{T} .
$$

Now, assume the theorem is true for $b+1$, i.e.,

$$
H_{2 n+b+1}={ }^{2 n-t} H_{1 \times m} \times K_{m \times m} \times A_{m \times m}^{b+1} \times{ }^{t} U_{1 \times m}^{T} .
$$

Replacing $n$ by $n-\frac{1}{2}$ in Equation (11) and reversing the steps of the proof of Lemma 1, we have

$$
H_{2 n+b}={ }^{2 n-t} H_{1 \times m} \times K_{m \times m} \times A_{m \times m}^{b} \times{ }^{t} U_{1 \times m}^{T} .
$$


Lemma 2. Let $m, n$ be positive integers. We have that

$$
H_{m+n}={ }^{m} H_{1 \times m} \times K_{m \times m} \times{ }^{n} U_{1 \times m}^{T}
$$

where ${ }^{n} U^{T}$ denotes the transpose of the matrix ${ }^{n} U$.

Proof. By Proposition 1, we have $H_{n}={ }^{(n-t)} H_{1 \times m} \times K_{m \times m} \times{ }^{t} U_{1 \times m}^{T}$ Now, by replacing $t$ by $n-m$, we get

$$
H_{n}={ }^{m} H_{1 \times m} \times K_{m \times m} \times{ }^{n-m} U_{1 \times m}^{T} .
$$

In particular, when $n$ is replaced by $n+m$, we have

$$
H_{m+n}={ }^{m} H_{1 \times m} \times K_{m \times m} \times{ }^{n} U_{1 \times m}^{T} .
$$

\subsection{Procedure}

In this subsection, we provide a method of finding the $n$th term of any linear homogeneous recurrence $m$-step relation using the last few terms of its corresponding Fibonacci's family $m$-step sequence. To find the $n$th term of the recurrence relation $H_{n}, n \geq 3 m$, we apply the following steps:

1. Find ${ }^{m} U$.

2. Compute $K \times A^{-t}$, where $1 \leq t \leq m-1$.

3. Set $z:=\left\lfloor\log _{2}(n-m)\right\rfloor$ and $w:=\left\lfloor\log _{2}\left(m+b_{1}\right)\right\rfloor$.

4. Rewrite $n-m$ as $\left(\left(\left(m+b_{1}\right) \times 2+b_{2}\right) \cdots \times 2+b_{z-w+1}\right)$, where $b_{i}=0$ or $1,2 \leq i \leq z-w+1$, and $0 \leq b_{1} \leq 2^{\left\lfloor\log _{2}(m)\right\rfloor+1}-m$.

5. Find ${ }^{m+b_{1}} U$.

6. Set $^{0} S:={ }^{m+b_{1}} U$.

7. Use the following algorithm to find ${ }^{z-w} S:={ }^{n-m} U$, for $i=1: z-w$ :

\{

$$
\begin{aligned}
& \text { for }(j=1: m) \text { : } \\
& \{ \\
& { }^{i} S(1, j)={ }^{i-1} S \times\left(K \times A^{1-j}\right) \times{ }^{i-1} S^{T} \\
& \text { \} } \\
& \text { If } b_{i+1}=1 \text { : }
\end{aligned}
$$

8. $H_{n}={ }^{m} H \times K \times{ }^{z-w} S^{T}$.

\subsection{Numerical Example}

Given $H_{n}=H_{n-1}-2 H_{n-2}+H_{n-3}-H_{n-4}+H_{n-6}+H_{n-7}$, with initial values

$$
H_{1}=1, H_{2}=2, H_{3}=1, H_{4}=4, H_{5}=-3, H_{6}=6, H_{7}=-4 \text {. }
$$


We use our previous procedure to compute $H_{46}$.

1. ${ }^{0} S:={ }^{m} U_{1 \times m}=\left[\begin{array}{lllllll}2 & 2 & 0 & -2 & -1 & 1 & 1\end{array}\right]$

2. $K_{m \times m}=\left(\begin{array}{ccccccc}1 & -2 & 1 & -1 & 0 & 1 & 1 \\ -2 & 1 & -1 & 0 & 1 & 1 & 0 \\ 1 & -1 & 0 & 1 & 1 & 0 & 0 \\ -1 & 0 & 1 & 1 & 0 & 0 & 0 \\ 0 & 1 & 1 & 0 & 0 & 0 & 0 \\ 1 & 1 & 0 & 0 & 0 & 0 & 0 \\ 1 & 0 & 0 & 0 & 0 & 0 & 0\end{array}\right), \quad A_{m \times m}=\left(\begin{array}{ccccccc}1 & -2 & 1 & -1 & 0 & 1 & 1 \\ 1 & 0 & 0 & 0 & 0 & 0 & 0 \\ 0 & 1 & 0 & 0 & 0 & 0 & 0 \\ 0 & 0 & 1 & 0 & 0 & 0 & 0 \\ 0 & 0 & 0 & 1 & 0 & 0 & 0 \\ 0 & 0 & 0 & 0 & 1 & 0 & 0 \\ 0 & 0 & 0 & 0 & 0 & 1 & 0\end{array}\right)$

3. $z=5, n-m=39=(((7+2) \times 2+1) \times 2+1), b_{1}=2, b_{2}=1, b_{3}=1$ and $w=3$.

4. $\quad{ }^{0} S:={ }^{9} U_{1 \times 7}=\left[\begin{array}{lllllll}0 & 2 & 2 & 2 & 0 & -2 & -1\end{array}\right]$

5. ${ }^{1} S=\left[\begin{array}{lllllll}0 & -28 & -28 & -12 & 12 & 20 & 5\end{array}\right]$

6. $b_{2}=1$, then

$$
\begin{aligned}
& { }^{1} S=\left[\begin{array}{lllllll}
65 & 0 & -28 & -28 & -12 & 12 & 20
\end{array}\right] \\
& { }^{2} S=\left[\begin{array}{lllllll}
11409 & 4897 & -2432 & -4792 & -2696 & 664 & 2312
\end{array}\right]
\end{aligned}
$$

7. $b_{3}=1$, then

$$
\begin{aligned}
{ }^{2} S & =\left[\begin{array}{rrrrrrr}
6951 & 11409 & 4897 & -2432 & -4792 & -2696 & 664
\end{array}\right] \\
\text { 8. } H_{46} & =30091 .
\end{aligned}
$$

\section{Application}

In this section, we present an application that is concerned with computational number theory. By Property 1 , we have $\lim _{n \rightarrow \infty} \frac{F_{n}}{F_{n-1}}=\frac{1+\sqrt{5}}{2}$, where $F_{n}$ is the classical Fibonacci sequence. Therefore, one can estimate $\sqrt{5}$ using two consecutive terms in the Fibonacci sequence.

It is well known that the explicit form of the recurrence relation $F_{n}=\left(r_{1}+r_{2}\right) F_{n-1}-\left(r_{1} r_{2}\right) F_{n-2}$ is given by

$$
F_{n}=c_{1} r_{1}^{n}+c_{2} r_{2}^{n}
$$

where $r_{1}, r_{2}$ are the roots of the characteristic equation $x^{2}-x-1=0$ and $c_{1}, c_{2}$ are constants. We use this fact to approximate imperfect square roots.

Let $a$ be an imperfect square. To approximate $\sqrt{a}$, rewrite $a$ as $(b+i)^{2} \times 10^{m}$, where $b \in Z, 0<$ $i<1,1 \leq b \leq 9$, and $\frac{m}{2} \in Z$. Since $\sqrt{a}=10^{m / 2} \times(b+i)$, we only need to find $b+i$.

Let $H_{n}=r_{1}^{n}+r_{2}^{n}$, where $r_{1}=b-(b+i)$ and $r_{2}=b+(b+i)$. Then $H_{n}=\left(r_{1}+r_{2}\right) H_{n-1}-$ $\left(r_{1} r_{2}\right) H_{n-2}=(2 b) H_{n-1}-\left(b^{2}-(b+i)^{2}\right) H_{n-2}$. Since $\lim _{n \rightarrow \infty} \frac{H_{n}}{H_{n-1}}=r_{2}=2 b+i$, then $\sqrt{a}=(b+i) \times$ $10^{m / 2} \approx\left(\frac{H_{n}}{H_{n-1}}-b\right) \times 10^{m / 2}$.

Author Contributions: I.N.J. conceived the presented idea. I.N.J developed the theory and performed the computations. M.R.A. verified the analytical methods. M.R.A. encouraged I.N.J. to investigate the relation between recurrence relations and their characteristic roots to find an application for theorem 1. M.R.A. encouraged I.N.J. to use matrix notation to proof theorem 1 and supervised the findings of this work. Both authors discussed the results and contributed to the final manuscript.

Funding: This research was funded by King Fahd University of Petroleum and Minerals, grant number USRG1702.

Acknowledgments: The authors would like to acknowledge the support provided by the Deanship of Scientific Research at King Fahd University of Petroleum and Minerals for funding this work through project No. USRG1702.

Conflicts of Interest: The authors declare no conflict of interest. 


\section{References}

1. Choi, E. The sum of $k$ distanced tribonacci numbers. Glob. J. Pure Appl. Math. 2016, 12, 4041-4055.

2. Choo, Y. On the generalizations of fibonacci identities. Results Math. 2017, 71, 347-356. [CrossRef]

3. Kaddoura, I.; Mourad, B. On a new improved unifying closed formula for all fibonacci-type sequences and some applications. J. Number Theory 2018, 182, 271-283. [CrossRef]

4. Miles, E.P. Generalized fibonacci numbers and associated matrices. Am. Math. Mon. 1960, 67, 745-752. [CrossRef]

5. Sapir, A.; Sapir, A.; Kogana, T.; Sapir, L. The fibonacci family of iterative processes for solving nonlinear equations. Appl. Numer. Math. 2016, 110, 148-158.

6. Natividad, L.R.; Policarpio, P.B. A novel formula in solving tribonacci-like sequence. Gen. Math. Notes 2013, 17, 82-87.

7. Singh, S.; Bhadouria, P.; Sikhwa, O.; Sisodiya, K. A formula for tetranacci-like sequence. Gen. Math. Notes 2014, 20, 136-141.

8. Rathore, G.R.; Sikhwal, O.; Choudhary, R. Formula for finding $n$th term of fibonacci-like sequence of higher order. Int. J. Math. Appl. 2016, 4, 75-80.

9. Miller, J.C.; Spencer Brown, D.J. An algorithm for evaluation of remote terms in a linear recurrence sequence. Comput. J. 1966, 9, 188-190. [CrossRef]

(C) 2019 by the authors. Licensee MDPI, Basel, Switzerland. This article is an open access article distributed under the terms and conditions of the Creative Commons Attribution (CC BY) license (http:/ / creativecommons.org/licenses/by/4.0/). 